\title{
A novel cross-sector telemedical approach to detect arrhythmia in primary care patients with palpitations using a patient-activated event recorder
}

\author{
Oliver Klein-Wiele ${ }^{1,2}$, Markus Faghih ${ }^{1}$, Stefan Dreesen ${ }^{1}$, Rhyan Urbien ${ }^{1}$, \\ Marwan Abdulghafor ${ }^{1}$, Kaffer Kara ${ }^{3}$, Michael Schulte-Hermes ${ }^{1}$, \\ Marietta Garmer ${ }^{2}$, Dietrich Grönemeyer ${ }^{2}$, Birgit Hailer ${ }^{1}$ \\ ${ }^{1}$ Department of Cardiology, University of Witten/Herdecke, Essen, Germany \\ ${ }^{2}$ Grönemeyer Institute, University of Witten/Herdecke, Essen, Germany \\ ${ }^{3}$ Department of Cardiology, University of Bochum, Bochum, Germany
}

\begin{abstract}
Background: Patient-activated event recorders (ER) can facilitate diagnosis in unclear palpitations, however impact of ER screening on further treatment in clinical routine is unknown. We investigated the feasibility and clinical value of a network-based telemetric monitoring using a patient activated ER.
\end{abstract}

Methods: The network consisted of 12 general practitioners (GP) and a department of cardiology (DC). GP-patients sent electrocardiograms (ECGs) twice daily and in case of palpitations. ECGs were transferred by email to GP and DC and analyzed independently by both. The therapeutic strategy was discussed between GP and DC. The monitoring period ended after 4 weeks or in case of detected arrhythmia.

Results: A group of 184 consecutive patients were retrospectively analyzed. Mean age was $57.5 \pm 14.4$ years (range 17-82), 104 (56.5\%) were female. Significant arrhythmia occurred in $71(38.5 \%)$ patients: Recurrence of known paroxysmal atrial fibrillation $(A F ; n=27,14.7 \%)$, de novo AF ( $n=19,10.3 \%)$, premature complexes/bigeminus ( $n=13,7.1 \%)$, sinus tachycardia $(n=7,3.8 \%)$, atrioventricular nodal reentrant tachycardia $(n=3,1.6 \%)$, and ventricular tachycardia $(n=2,1.1 \%)$. A therapeutic consequence resulted in $63(88.7 \%)$ patients with detected arrhythmia: new oral anticoagulation $(n=29,40.8 \%)$, new antiarrhythmic medication ( $n=27,38.0 \%)$, behavioral intervention $(n=19,26.8 \%)$, electrophysiology-study/catheter ablation $(n=4,5.6 \%)$, cardioversion $(n=2,2.8 \%)$, implantable cardioverter-defibrillator-implantation ( $n=1,1.4 \%)$, and left atrial appendage occluder $(n=1,1.4 \%)$.

Conclusions: The investigated cross-sector telemetric network is a feasible approach to detect arrhythmia in patients with palpitations and may have high impact on further treatment, notably in those at risk for stroke due to AF. (Cardiol J 2016; 23, 4: 422-428)

Key words: event recorder, atrial fibrillation, palpitations, general practice, telemedicine, telecardiology

Address for correspondence: Dr. Oliver Klein-Wiele, University of Witten/Herdecke, Department of Cardiology, Katholisches Klinikum Essen, Hülsmannstraße 17, 45355 Essen, Germany, tel: +49 2016400 3301/+49 178 5382453 , fax: +492016400 3304, e-mail: o.klein-wiele@kk-essen.de

Received: 19.03.2016

Accepted: 06.06.2016 


\section{Introduction}

Palpitations suggestive of cardiac arrhythmia are a common symptom in outpatients seeking medical attendance [1]. Less than a half of those patients suffer from cardiac arrhythmia [2]. A 12-lead electrocardiogram (ECG) during symptoms as the gold standard of defining arrhythmia in symptomatic patients can be obtained only in about 30\% [3]. Due to the transient nature of most arrhythmias, detection can be challenging. From $24 \mathrm{~h}$ to $48 \mathrm{~h}$ Holter ECG is still the standard of care for these patients, nevertheless it has a low diagnostic yield [4]. Implantable loop recorders are the gold standard of prolonged ambulant rhythm monitoring [5]. However, their use is restricted due to high costs, invasiveness with up to $1 \%$ of pocket infections and cosmetic aspects [6]. Patient-activated event recorders (ER) have shown to be useful in this clinical setting. A higher diagnostic value compared to standard care with a higher number of relevant diagnoses has been reported in several studies as reviewed by Hoefman et al. [7]. Most of these trials have been conducted in tertiary care settings. In studies with primary care patients, ECGs were analyzed by external cardiologists. This appears to be necessary due to possible lack of expertise in detecting arrhythmias, such as atrial fibrillation (AF) by general practitioners (GP) [8]. However, external review of ECGs does not reflect the usual clinical routine. No data about the impact of ERscreening on further therapy in clinical practice exist. Hence, there is insufficient information about the therapeutic impact of these devices in a real-world setting in primary care. The aim of this study was to assess the diagnostic value and therapeutic impact of ERs in a newly established cross-sector telemedical network.

\section{Methods}

The study complies with the Declaration of Helsinki and was approved by the Ethics Committee of the University Witten/Herdecke.

\section{Description of the network}

Twelve GPs were invited to take part in the network and were provided with 2 ERs each. The GPs were instructed to apply ER monitoring in patients in sinus rhythm presenting with infrequent palpitations. The inclusion criteria were: palpitation (defined as the perception of a fast and/ /or irregular heartbeat) appearing at least once in 4 weeks and non-diagnostic resting ECG and $24 \mathrm{~h}$
Holter monitoring. The exclusion criteria were: less frequent symptoms, presence of implanted rhythm devices and patient's disability to record and transmit ECGs due to cognitive or physical impairment. The GP obtained informed consent and explained the use of the device. When a patient was selected for monitoring the GP sent a fax to the department of cardiology (DC) with the patient's data, clinical characteristics, and the serial number of the device. After 1 successful test transmission, patients were instructed to record and transfer ECGs twice daily and in case of palpitations. These daily routine ECG recordings intended to screen for asymptomatic AF episodes. In case of missing ECG transferals, the patients were contacted by phone by the GP staff. Technical issues and comprehension problems were solved by phone or in an additional practice appointment. In the DC, 5 experienced cardiologists analyzed the incoming ECGs daily. In case of detected arrhythmia, GP and DC discussed the further diagnostic and therapeutic approach by phone. The monitoring period ended after 4 weeks or in case of detected arrhythmia. A 4-week monitoring period was chosen for an optimum sensitivity of arrhythmia detection in patients with infrequent symptoms. Longer telemetry periods did not show additional benefit $[3,9]$.

\section{Technical characteristics}

In all cases, a Tele-EKG-Card $100 \mathrm{IR}^{\mathrm{TM}}$ (Vitaphone, Mannheim, Germany) was used (Fig. 1). This card consists of a memory unit that can store up to three $30 \mathrm{~s}$ single lead ECGs. Patients have to wear the device around the neck. In case of palpitations, the ECG card is pressed to the precordial region and activated by a button. Four fixed metal electrodes on the back of the device conduct signal transfer. Due to absent adhesive electrodes, there is no permanent electrical contact, i.e. there is no looping memory function. Guided by voice commands the patient sends the recorded acoustically encoded episodes to the Vitaphone server via a toll free number. Data is transformed to PDF-ECG files and transmitted to the clients via email. ECGs were assigned to an individual patient by the serial number of the device. Further information regarding symptoms was not specified in order to simplify the screening.

\section{Follow-up}

Monitoring results and the subsequent treatment were documented by the GP until the end of follow-up. Six months after the end of the last 


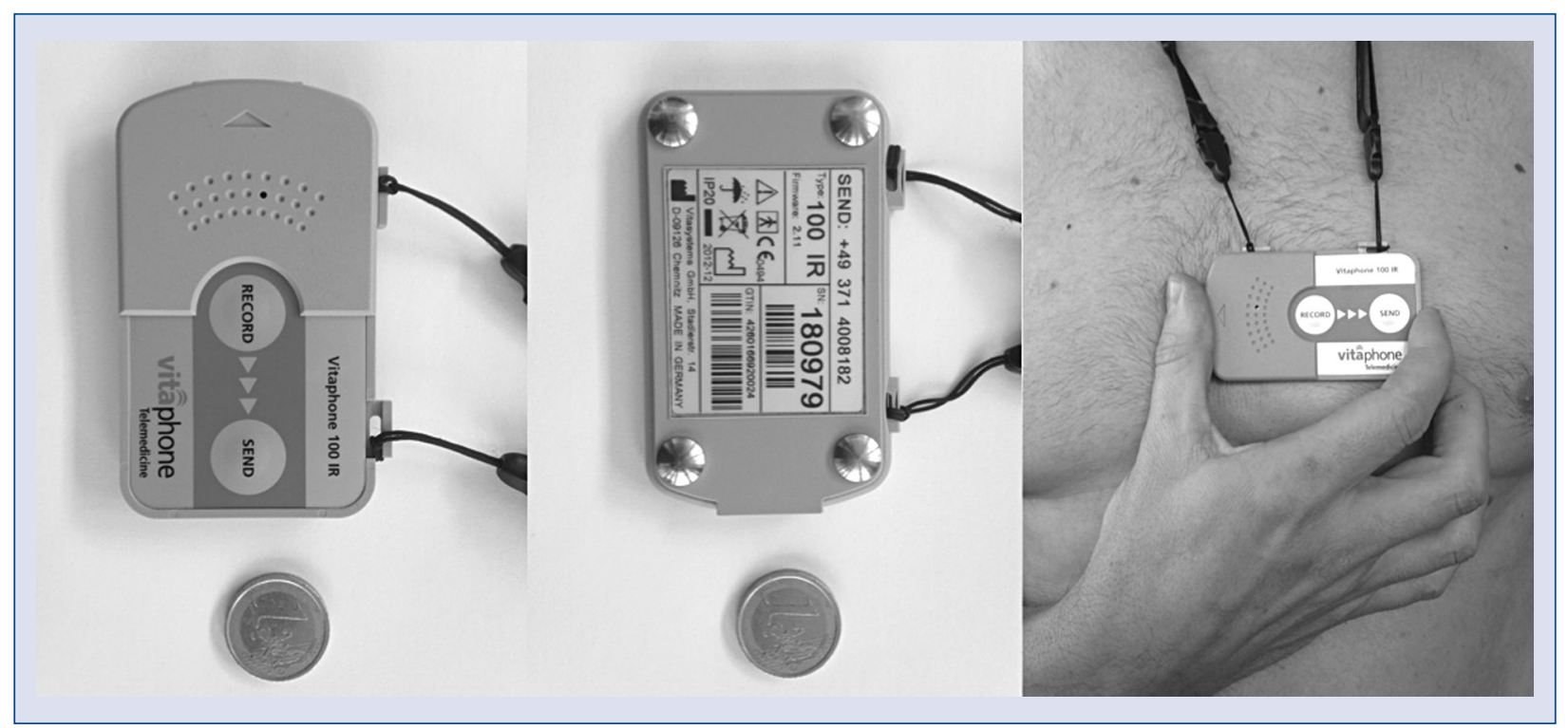

Figure 1. Tele-EKG-Card 100IR ${ }^{\mathrm{TM}}$ (Vitaphone, Mannheim, Germany).

patient's monitoring period the GP's patient records were reviewed for diagnostic results and the following therapeutic interventions based on ER monitoring: new or altered medical treatment (antiarrhythmics and anticoagulation), cardioversion, electrophysiology-study/catheter ablation, device therapy, left atrial appendage (LAA) occlusion, and behavioral intervention.

\section{Results}

\section{Diagnoses}

From October 2012 to July 2014, 184 patients with mean age $57.5 \pm 14.4$ years (range $17-82$ ), were monitored, $104(56.5 \%)$ were female (for general characteristics see Table 1). There were no dropouts due to technical or compliance problems. The device has no quality feedback function to reassure patients about the success of ECG recordings. Nevertheless, insufficient signal quality due to lack of electrical contact or motion artifacts was seen only in single ECGs. One hundred and thirteen $(61.4 \%)$ patients had no arrhythmic event. The majority of arrhythmic patients had recurrence of known paroxysmal $\mathrm{AF}(\mathrm{PAF})(\mathrm{n}=27$, $14.7 \%) ; 17(9.2 \%)$ with $\mathrm{CHA}_{2} \mathrm{DS}_{2} \mathrm{VASc}$ score $>1$; followed by de novo $\mathrm{AF}(\mathrm{n}=19,10.3 \%) ; 12(6.5 \%)$ with $\mathrm{CHA}_{2} \mathrm{DS}_{2} \mathrm{VASc}$ score $>1$. Atrioventricular nodal reentrant tachycardia (AVNRT) was found in $3(1.6 \%)$ patients. Sustained and non-sustained ventricular tachycardia was detected in 1 patient, respectively. Less relevant arrhythmia, such as sinus tachycardia and premature complexes, was found in $20(10.9 \%)$ patients. None of the arrhythmic patients reported symptoms of circulatory compromise. Findings on ER monitoring are summarized in Table 2. Figure 2 shows examples of pathologic single lead-ECG.

\section{Therapeutic consequences}

Oral anticoagulation (OAC) was started in 14 out of $19(73.7 \%)$ patients with de novo AF and in 15 out of $27(55.5 \%)$ patients with recurrence of known PAF. This corresponds to new OAC in $15.8 \%$ based on all patients studied. The remaining were already anticoagulated, had $\mathrm{CHA}_{2} \mathrm{DS}_{2}$ VASc scores $\leq 1$ or refused OAC. In 7 of newly anticoagulated patients, direct OACs were given instead of vitamin $\mathrm{K}$ antagonists (VKA) after agreement between DC and GP. Of $46 \mathrm{AF}$ patients 2 (1 with known PAF, 1 with new $\mathrm{AF}$ ) were found to have persistent $\mathrm{AF}$; cardioversion was successfully performed after transesophageal echocardiography. In 1 patient with recurrence of PAF and history of gastrointestinal bleeding under VKA treatment as a result of esophagus varices due to liver cirrhosis and no $\mathrm{OAC}$ at the time of monitoring LAA occlusion was performed. In two highly symptomatic patients ( 1 with known PAF, 1 with new AF), sinus rhythm could be restored by pulmonary vein isolation. Three patients had AVNRT. Two of them had electrophysiology study and catheter ablation, 1 was treated medically due to young age and less severe symptoms. In 1 patient, detection 
Table 1. Baseline characteristics (total patients: $\mathrm{n}=184$ ).

\begin{tabular}{lcc}
\hline & Mean or N & Range or \% \\
\hline Age [years] & $57.5 \pm 14.4$ & $17-82$ \\
CHA $_{2}$ DS $_{2}$ VASc score & $1.9 \pm 1.4$ & $0-6$ \\
Female & 104 & 56.5 \\
Age groups [years]: & & \\
$\quad<50$ & 55 & 29.9 \\
$50-64$ & 62 & 33.7 \\
$65-75$ & 52 & 28.3 \\
$>75$ & 15 & 8.2 \\
Congestive heart failure & 6 & 3.3 \\
Hypertension & 103 & 56.0 \\
Diabetes mellitus & 16 & 8.7 \\
Previous stroke & 10 & 5.4 \\
Coronary artery disease & 21 & 11.4 \\
Known paroxysmal AF: & 52 & 28.3 \\
CHA ${ }_{2}$ DS ${ }_{2}$ VASc $>1$ & 35 & 19.0 \\
Without pre-existing & 26 & 14.1 \\
OAC & & \\
CHA & \\
without ${ }_{2}$ VASc $>1$ & 18 & 9.7 \\
OAC & & \\
\hline
\end{tabular}

$\mathrm{AF}$ - atrial fibrillation; $\mathrm{OAC}$ - oral anticoagulation

Table 2. Findings on event recorder monitoring.

\begin{tabular}{lcc}
\hline & N & $\%$ \\
\hline No arrhythmia (sinus rhythm) & 113 & 61.4 \\
Relevant arrhythmia & 71 & 38.6 \\
Recurrence of AF: & 27 & 14.7 \\
$\mathrm{CHA}_{2} \mathrm{DS}_{2}$ VASc $>1$ & 17 & 9.2 \\
Without pre-existing OAC $_{\mathrm{CHA}_{2} \mathrm{DS}_{2} \text { VASc }>1}$ & 16 & 8.7 \\
without pre-existing OAC $_{\text {New AF: }}$ & 11 & 6.0 \\
$\quad \mathrm{CHA}_{2} \mathrm{DS}{ }_{2}$ VASc $>1$ & 19 & 10.3 \\
Premature complexes/bigeminus & 12 & 6.5 \\
Sinus tachycardia & 7 & 7.1 \\
AVNRT & 3 & 3.8 \\
Sustained VT & 1 & 0.5 \\
Non-sustained VT & 1 & 0.5 \\
\hline
\end{tabular}

AF - atrial fibrillation; AVNRT - atrioventricular nodal reentrant tachycardia; OAC — oral anticoagulation; VT — ventricular tachycardia

of a sustained ventricular tachycardia resulted in further cardiologic evaluation. Implantation of an implantable cardioverter-defibrillator (ICD) was performed after dilative cardiomyopathy was diagnosed. In a 25 -year-old female patient, recurrent non-sustained ventricular tachycardia was found. Structural heart disease was excluded, betablocker medication was started resulting in relief of symptoms. Antiarrhythmic medication was started in a total of $27(38.0 \%)$ patients. In 5 of 20 patients with less relevant arrhythmia (sinus tachycardia, bigeminus, premature complexes), antiarrhythmic therapy with beta-blockers was initiated, 19 had short behavioral intervention which encompassed a 20-min educational conversation about the benign nature of the detected arrhythmia and possible coping strategies.

In ER negative patients and in the group with less relevant arrhythmia, there were no cardiologic referrals or hospital admissions, and neither in- nor outpatient emergency treatments on follow-up. Table 3 summarizes therapeutic consequences in patients with detected arrhythmia.

\section{Discussion}

Palpitations are a common symptom in general practice. GP can rarely establish a diagnosis in the case of infrequent palpitations due to the low sensitivity of $24 \mathrm{~h}$ Holter monitoring. This diagnostic dilemma leads to a significant number of hospital admissions, emergency treatments, and cardiologic referrals.

The present study shows that patient-triggered event recorders can be effectively integrated in the routine workup of patients with unclear palpitations in a cross-sector telemedical network.

The approach was suitable for the detection of $\mathrm{AF}$, which is the most common clinically relevant arrhythmia in the general population. The clinical impact of $\mathrm{AF}$ is high: AF leads to a threefold increased risk of developing heart failure; risk of stroke is 5 -fold increased, and doubled mortality rates have been described [10]. Stroke due to $\mathrm{AF}$ leads to death or severe disability in over $70 \%$ cases [11]. Recent research on cryptogenic stroke has highlighted silent AF as a major cause of stroke [12].

Our study indicates that despite the generally benign character and excellent short-term prognosis [2] of palpitations, screening for AF exceeding standard workup is warranted, particularly in patients with $\mathrm{CHA}_{2} \mathrm{DS}_{2}$ VASc-score $>1$ in order to rule out $\mathrm{AF}$ as a cause of palpitations [13].

Although OACs can lower the risk of stroke due to $\mathrm{AF}$ by over $60 \%$ and mortality rates by $25 \%$ [14], they can only be used in documented AF. 


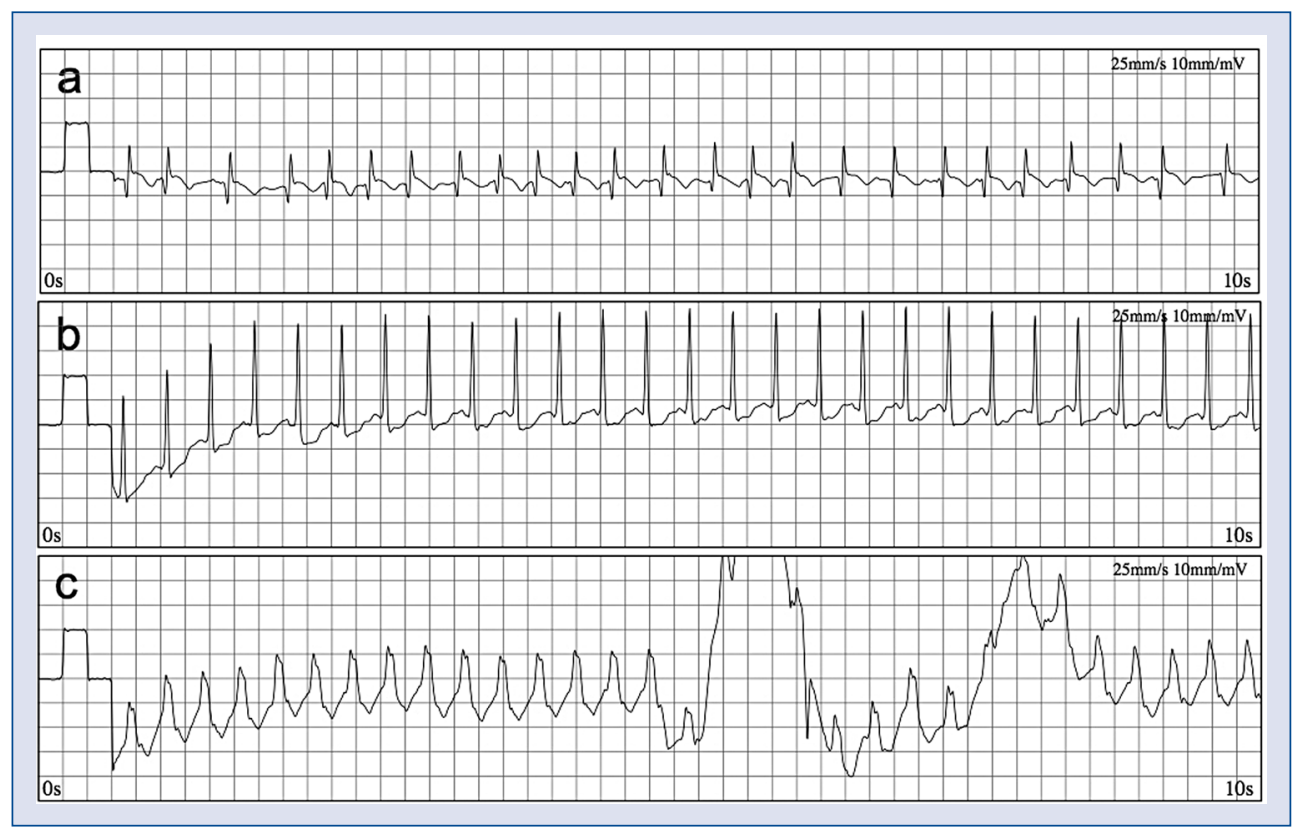

Figure 2. Examples of pathologic findings on transmitted single lead - electrocardiograms (ECGs). Only the first $10 \mathrm{~s}$ of the $30 \mathrm{~s}$ - ECG strip are displayed; A. Atrial fibrillation; B. Atrioventricular nodal reentrant tachycardia; C. Ventricular tachycardia.

Table 3. Therapeutic consequences in patients with detected arrhythmia (total: $\mathrm{n}=71^{*}$ ).

\begin{tabular}{lcc}
\hline & N & $\%{ }^{*}$ \\
\hline Any therapeutic consequence & 63 & 88.7 \\
New oral anticoagulation: & 29 & 40.8 \\
$\quad$ Known paroxysmal AF & 15 & 21.1 \\
Known paroxysmal AF & 11 & 15.5 \\
$\mathrm{CHA}_{2} \mathrm{DS}_{2} \mathrm{VASc}>1$ & & \\
De novo AF & 14 & 19.7 \\
De novo AF $\mathrm{CHA}_{2} \mathrm{DS}_{2} \mathrm{VASc}>1$ & 12 & 16.9 \\
VKA & 22 & 31.0 \\
$\quad$ DOAC & 7 & 9.9 \\
Antiarrhythmic medication & 27 & 38.0 \\
Behavioral intervention & 19 & 26.8 \\
Electrophysiology-study/ & 4 & 5.6 \\
/catheter ablation & & \\
Cardioversion & 2 & 2.8 \\
LAA-occluder & 1 & 1.4 \\
ICD implantation & 1 & 1.4 \\
\hline
\end{tabular}

*Multiple therapeutic consequences in a number of patients generate a sum of percentages $>100 \%$. AF - atrial fibrillation; VKA vitamin $\mathrm{K}$ antagonist: DOAC - direct oral anticoagulant; LAA left atrial appendage; ICD - implantable cardioverter-defibrillator

Being often asymptomatic and transient, AF mostly evades detection by $24 \mathrm{~h}$ Holter ECG. Studies showing that prolonged screening for AF is effec- tive [15] had little influence on clinical routine. This may be due to insufficient awareness of the problem in daily clinical practice and the lack of a practicable diagnostic approach in primary care. Integrated in a cross-sector telemedical network, the patient-activated ER is applicable for routine use in outpatients with suspected PAF. Combining symptom-triggered and routine ECG-transmission for palpitations appears to be effective in the detection of AF and it is well accepted by the patients.

The clinical impact of the established diagnoses was high, notably case discussion between GP and DC led to $100 \%(12 / 12)$ anticoagulation rate in de novo $\mathrm{AF}$ patients with a $\mathrm{CHA}_{2} \mathrm{DS}_{2} \mathrm{VASc}$ score $>1$ on follow-up.

There is evidence that auto-triggered detection of arrhythmia may be more effective in diagnosing relevant arrhythmia, particularly AF, than the patient-triggered mode alone [16]. However, adhesive electrodes are necessary, which can be inconvenient when used over a long period of time. We used a device without a looping memory to improve patient compliance and convenience and combined symptom-triggered monitoring with routine ECG transferal twice daily to screen for asymptomatic AF. This approach has been successfully employed in previous trials on posttherapeutic recurrence of AF: the device was equally effective as 7-day Holter monitoring [17] 
and is supported by the European Heart Rhythm Association guideline on indications for diagnostic loop recorders [5]. In a recently published STUDY-AF trial [18], an external continuous loop recorder with auto-detection of AF was used in asymptomatic patients with the following AF risk factors: coronary heart disease, heart failure, hypertension, diabetes mellitus, and sleep apnea to screen for silent AF. We could show a higher detection rate ( $5.3 \%$ vs. $10.3 \%$ ) of de novo AF. This indicates that the presence of palpitations may correlate with a higher rate of detected $\mathrm{AF}$ in patients at risk for $\mathrm{AF}$ and its sequelae. Another study [19] investigated patients with palpitations using a device similar to the Tele-EKG-Card 100IR ${ }^{\mathrm{TM}}$ and found only $2.6 \%$ of not further specified atrial tachycardia. Monitoring was limited to 15 days. This supports the finding that a monitoring period of 4 weeks is preferable [3].

Event recorder screening was not limited to patients without previously diagnosed arrhythmia. Thus, patients with known PAF were also screened. Interestingly, only a half of 52 patients with known PAF and palpitations showed recurrence of $\mathrm{AF}$ in the monitoring period. We conclude that palpitations in PAF patients should not generally be attributed to PAF. One of those patients had sustained ventricular tachycardia requiring ICD implantation; most were in sinus rhythm. Furthermore, screening patients with known PAF can also be of relevance regarding anticoagulation management. Many AF patients are not treated with OAC despite a $\mathrm{CHA}_{2} \mathrm{DS}_{2} \mathrm{VASc}$ score $>1$ and missing contraindications [20]. In our study, 18 (51.4\%) patients with known PAF and a $\mathrm{CHA}_{2}-$ $\mathrm{DS}_{2}$ VASc score $>1(\mathrm{n}=35,67.3 \%)$ did not receive OAC. Eleven $(61.1 \%)$ of them had AF on monitoring. Detection of recurrent PAF led to $100 \%$ anticoagulation rate in ER positive patients with a $\mathrm{CHA}_{2} \mathrm{DS}_{2} \mathrm{VASc}$ score $>1$ and no OAC prior to ER-screening. Thus, discussion between GP and DC may be beneficial for better implementation of guideline conform therapy. Patients without arrhythmia on ER-screening were not subject to discussion between GP and DC; accordingly, also patients with known PAF that did not show recurrence of PAF on screening. Nine of these patients had a $\mathrm{CHA}_{2} \mathrm{DS}_{2}$ VASc score $>1$. Case discussion might have been beneficial also in these patients because ER screening cannot exclude recurrence of $\mathrm{AF}$ in $100 \%$.

The network approach provides an efficient communication between GP and DC and fast clinical decision-making. This link between GP and $\mathrm{DC}$ has special significance for AF-patients. Decisions about anticoagulation have to take into account compliance issues, risk of falls, and bleeding complications. GPs have more insight in daily life aspects and in the morbidity of their patients. On the other hand, cardiologic expertise is needed to decide what strategy (rate vs. rhythm control/ /anticoagulation vs. LAA-occluder) is most suitable. The network is a good platform for structured communication about rhythm management and allows for an individualized approach, minimizing loss of information. This is reflected by a high over all number of initiated treatments.

Despite absence of severe arrhythmia, life can be impaired due to the recurrence of symptoms and insecurity in patients with unclear palpitations leading to hospitalizations or intensified diagnostic efforts. We could show that ruling out severe arrhythmia in the monitoring period may be helpful in limiting further diagnostic efforts: during 6-month follow-up, there were no planned hospital admissions, cardiologic referrals, or emergency treatments of those patients.

Although failure of device activation or ECG-transmission by the patient can occur [21], this was an irrelevant obstacle in the present trial, mainly because patients were contacted by the GP staff in the case of missing routine ECG transmission.

The present study shows that network-based ER-monitoring can be a useful part in a graduated diagnostic approach to palpitations closing the gap between standard $24 \mathrm{~h}$ Holter monitoring and more cost intensive and invasive implantable loop recorders.

\section{Limitations of the study}

One major limitation of this study is the missing control group. Secondly, excluded patients were not registered and, therefore, could not be analyzed. We encourage controlled prospectiverandomized trials in order to clarify the clinical significance of our results. To simplify the approach, symptoms during monitoring were not documented. Therefore, arrhythmic episodes could not be attributed as symptomatic or asymptomatic. However, the intention of the network to exclude prognostically relevant arrhythmia is unaffected by this issue. Screening for AF with ECG monitoring twice daily cannot exclude AF by $100 \%$; the screening method has a sensitivity of about $70 \%$ [5]. Implantable loop recorders are the gold standard regarding AF detection. However, implantable loop recorders implantation appears to be no reasonable first-line approach for screening in the group of patients studied. 
There was no general screening for structural heart disease prior to inclusion. In order to keep our approach simple and cost efficient, this limitation may be acceptable and reflects general clinical practice.

\section{Conclusions}

The investigated cross-sector telemetric network using a non-invasive ER is a feasible approach to detect arrhythmia in patients with palpitations and may have high impact on further treatment, notably in those at risk for stroke due to $\mathrm{AF}$.

\section{Acknowledgements}

We acknowledge Dr. M. Busch for revising the manuscript.

\section{Conflict of interest: None declared}

\section{References}

1. Zimetbaum P, Josephson ME. Evaluation of patients with palpitations. N Engl J Med, 1998; 338: 1369-1373.

2. Weber BE, Kapoor WN. Evaluation and outcomes of patients with palpitations. Am J Med, 1996; 100: 138-148.

3. Hoefman E, van Weert HC, Boer KR, Reitsma J, Koster RW, Bindels PJ. Optimal duration of event recording for diagnosis of arrhythmias in patients with palpitations and light-headedness in the general practice. Fam Pract, 2007; 24: 11-13.

4. Hoefman E, van Weert HC, Reitsma JB, Koster RW, Bindels PJ. Diagnostic yield of patient-activated loop recorders for detecting heart rhythm abnormalities in general practice: A randomised clinical trial. Fam Pract, 2005; 22: 478-484.

5. Brignole M, Vardas P, Hoffman E et al. Indications for the use of diagnostic implantable and external ECG loop recorders. Europace, 2009; 11: 671-687.

6. Brignole M, Sutton R, Menozzi C et al. Early application of an implantable loop recorder allows effective specific therapy in patients with recurrent suspected neurally mediated syncope. Eur Heart J, 2006; 27: 1085-1092.

7. Hoefman E, Bindels PJ, van Weert HC. Efficacy of diagnostic tools for detecting cardiac arrhythmias: Systematic literature search. Neth Heart J, 2010; 18: 543-551.

8. Mant J, Fitzmaurice DA, Hobbs FD et al. Accuracy of diagnosing atrial fibrillation on electrocardiogram by primary care practition- ers and interpretative diagnostic software: Analysis of data from screening for atrial fibrillation in the elderly (SAFE) trial. BMJ, 2007; 335: 380 .

9. Attanasio P, Huemer M, Loehr L et al. Use of a patient-activated event recording system in patients with tachycardic palpitations: How long to follow up? Ann Noninvasive Electrocardiol, 2015; 20: 566-569.

10. Benjamin EJ, Wolf PA, D‘Agostino RB, Silbershatz H, Kannel WB, Levy D. Impact of atrial fibrillation on the risk of death: The Framingham Heart Study. Circulation, 1998; 98: 946-952.

11. Saposnik G, Gladstone D, Raptis R, Zhou L, Hart RG, Investigators of the RCSN and the SORCan Working Group. Atrial fibrillation in ischemic stroke: predicting response to thrombolysis and clinical outcomes. Stroke, 2013; 44: 99-104.

12. Gladstone DJ, Spring M, Dorian P et al. Atrial fibrillation in patients with cryptogenic stroke. N Engl J Med, 2014; 370: 2467-2477.

13. Lip GY, Halperin JL. Improving stroke risk stratification in atrial fibrillation. Am J Med, 2010; 123: 484-488.

14. Hart RG, Pearce LA, Aguilar MI. Meta-analysis: Antithrombotic therapy to prevent stroke in patients who have nonvalvular atrial fibrillation. Ann Intern Med, 2007; 146: 857-867.

15. Kishore A, Vail A, Majid A et al. Detection of atrial fibrillation after ischemic stroke or transient ischemic attack: a systematic review and meta-analysis. Stroke, 2014; 45: 520-526.

16. Balmelli N, Naegeli B, Bertel O. Diagnostic yield of automatic and patient-triggered ambulatory cardiac event recording in the evaluation of patients with palpitations, dizziness, or syncope. Clin Cardiol, 2003; 26: 173-176.

17. Piorkowski C, Kottkamp H, Tanner $\mathrm{H}$ et al. Value of different follow-up strategies to assess the efficacy of circumferential pulmonary vein ablation for the curative treatment of atrial fibrillation. J Cardiovasc Electrophysiol, 2005; 16: 1286-1292.

18. Turakhia MP, Ullal AJ, Hoang DD et al. Feasibility of extended ambulatory electrocardiogram monitoring to identify silent atrial fibrillation in high-risk patients: The Screening Study for Undiagnosed Atrial Fibrillation (STUDY-AF). Clin Cardiol, 2015; 38: 285-292.

19. de Asmundis C, Conte G, Sieira J et al. Comparison of the patient-activated event recording system vs. traditional $24 \mathrm{~h}$ Holter electrocardiography in individuals with paroxysmal palpitations or dizziness. Europace, 2014; 16: 1231-1235.

20. Buckingham TA, Hatala R. Anticoagulants for atrial fibrillation: why is the treatment rate so low? Clin Cardiol, 2002; 25: $447-454$.

21. Gula LJ, Krahn AD, Massel D, Skanes A, Yee R, Klein GJ. External loop recorders: determinants of diagnostic yield in patients with syncope. Am Heart J, 2004; 147: 644-648. 